\title{
Correlation between domain structure, surface anisotropy and high frequency magneto-impedance in Joule annealed CoFe-based melt-spun ribbons
}

\author{
T. Eggers ${ }^{1}$, A. Leary ${ }^{2}$, M. McHenry ${ }^{2}$, J. Marcin ${ }^{3}$, I. Škorvánek ${ }^{3}$, H. Srikanth ${ }^{1}$, M.H. Phan ${ }^{1, *}$ \\ ${ }^{1}$ Department of Physics, University of South Florida, Tampa, FL 33620, USA \\ ${ }^{2}$ Materials Science and Engineering Department, Carnegie Mellon University, Pittsburgh, \\ PA 15213, USA \\ ${ }^{3}$ Institute of Experimental Physics, Slovak Academy of Sciences, Watsonova 47, 04001 \\ Kosice, Slovakia
}

$\left(\mathrm{Fe}_{65} \mathrm{Co}_{35}\right)_{83.5-\mathrm{x}} \mathrm{B}_{13} \mathrm{Nb}_{\mathrm{x}} \mathrm{Si}_{2} \mathrm{Cu}_{1.5}$ amorphous ribbons with (4 at. \%) and without (0 at. \%) growth inhibitor $\mathrm{Nb}$ were Joule annealed at low current intensity to avoid bulk crystallization, and their surface domain structure, bulk and surface magnetization, and high frequency magneto-impedance (MI) were systematically investigated. Magneto-optic Kerr effect (MOKE) microscopy and longitudinal MOKE evidenced the development of a longitudinal surface anisotropy in the ribbon without $\mathrm{Nb}(\mathrm{Nb} 0)$ as a result of the Joule annealing treatment, while the 4 at. \% $\mathrm{Nb}(\mathrm{Nb} 4)$ ribbon developed an anisotropy $30^{\circ}$ from the transverse direction. The increased transverse ac permeability at the surface of $\mathrm{Nb} 4$ was evident in MI measurements by a higher $[\Delta Z / Z]_{\max }$ as compared to $\mathrm{Nb} 0$ at frequencies greater than $200 \mathrm{MHz}$. A correlation between the surface domain structure, induced anisotropy and high frequency MI in the ribbons was established.

PACS: $75.50 . \mathrm{Kj}$,

Keywords: Fe-rich amorphous ribbons; Surface anisotropy; Magneto-impedance; Magnetic sensors

*Corresponding authors: sharihar@usf.edu; phanm@usf.edu 


\section{Introduction}

The discovery [1] of a giant magneto-impedance (GMI) response in soft ferromagnetic amorphous ribbons and wires has spurred much study over the past 20 years due to exciting opportunities for its use as sensitive magnetic field sensors for a variety of applications [2]. The first groups to consider GMI for technological application were chiefly concerned with sensitive changes in electrical impedance at excitation frequencies less than $10 \mathrm{MHz}[3,4]$. They described the impedance change as the classical consequence of the skin effect, where the distribution of an ac current of frequency, $f$, in a soft magnetic conductor of conductivity, $\sigma$, flows near the surface at a depth $\delta=\left(\pi \sigma f \mu_{T}\right)^{-1 / 2}$. The large and sensitive transverse magnetic permeability, $\mu_{T}$, of soft ferromagnetic conductors is the key to unlocking giant changes in impedance. Since its discovery, magneto-impedance (MI) has become a common tool used to probe the ac transverse permeability of many magnetic materials [5-7].

FeCo alloys have previously been studied for their use in power conversion applications which utilize their excellent bulk magnetic properties of high saturation magnetization and magnetic softness $[8,9]$. The high permeability of these alloys also makes them interesting from the standpoint of high-frequency MI, where small magnetic fields can induce large variations in transverse permeability, thus large changes in impedance through the skin depth [10]. At excitation frequencies greater than $100 \mathrm{MHz}$, the skin depth becomes relatively small, $\delta \sim 0.5-4 \mu \mathrm{m}$, depending upon resistivity and permeability values of a material [11]. Therefore, considering the magnetic behavior of the surface is essential for interpreting the MI response at high frequency.

Melt-spun Fe-rich FeCo ribbons, such as those in this study, are amorphous and have quenched-in stresses that are a dominant source of their magnetic anisotropy. The as-quenched domain configuration is generally inhomogeneous over the ribbon surface and thickness, making 
the large distribution of magnetic anisotropy in ribbons unfavorable for high-frequency sensor applications. Thermomagnetic treatments such as transverse magnetic field annealing (TMF) can nucleate nanocrystalline phases within the amorphous matrix, providing a source for well-defined magnetocrystalline anisotropy in the bulk ribbon $[11,12]$. Previous work using TMF has found that the induced anisotropy of the ribbon can be controlled through nanocrystallization by adding $\mathrm{Nb}$ as a growth inhibitor, which suppresses crystallite size and increases bulk permeability values [13-15]. While annealing treatments succeed at reducing the random anisotropy distribution caused by internal stresses trapped by rapid solidification, bulk crystallization of FeCo-based ribbons causes embrittlement, which makes the ribbon difficult to realize in applications [8]. Besides TMF annealing, Joule annealing (JA) by application of a dc current also has the benefit of an applied magnetic field during the thermal treatment. With low current intensities and long annealing times, it is possible to relax the frozen-in stresses via interatomic diffusion while staying below the crystallization temperature. The magnetic field created by the annealing current has the potential to act as the driving force for interatomic diffusion, where it can interact locally with the magnetization of a microscopic region (a domain or grain or crystallite) [16]. Upon cooling, however, the current is shut off and the magnetic field disappears. Thus, the magnetic structure set up by the guided diffusion could deteriorate.

In this work, the surface domain structure, surface anisotropy and high frequency magnetotransport of Fe-rich FeCo ribbons with (4 at. \%) and without (0 at. \%) growth inhibitor $\mathrm{Nb}$ are examined. The as-cast ribbons were Joule annealed at low current amplitudes to see how the growth inhibitor influences the surface anisotropy when bulk crystallization is intentionally avoided. Surface sensitive techniques like magneto-optical Kerr effect and high-frequency magnetoimpedance were employed to determine the effect of the $\mathrm{Nb}$ addition on the surface magnetic properties of the ribbons before and after annealing. The results obtained provide important 
understandings of the role of growth inhibitor $\mathrm{Nb}$ in Fe-rich FeCo ribbons in regard to their prospective use in high frequency MI-based sensors.

\section{Materials and Methods}

Amorphous ribbons of composition $\left(\mathrm{Fe}_{65} \mathrm{Co}_{35}\right)_{83.5-\mathrm{x}} \mathrm{B}_{13} \mathrm{Nb}_{\mathrm{x}} \mathrm{Si}_{2} \mathrm{Cu}_{1.5}(x=0$ and 4$)$ (denoted throughout this work as $\mathrm{Nb0}$ and $\mathrm{Nb} 4$, respectively) were prepared by melt-spinning [14]. The thickness of the ribbons was $30 \mu \mathrm{m}$ and they were $2 \mathrm{~mm}$ wide. The ribbons were cast in very long strands from which $4 \mathrm{~cm}$ sections were cut and subject to a dc Joule heating treatment of $500 \mathrm{~mA}$ $\left(8.3 \mathrm{~A} / \mathrm{mm}^{2}\right)$ for $3 \mathrm{~h}$. At this current density, the steady state temperature measured by thermocouple on the surface of the ribbons reached $80{ }^{\circ} \mathrm{C}$. Due to high radiative losses at the ribbon surface, the annealing temperature is expected to be higher than $80{ }^{\circ} \mathrm{C}$, but still much less than the crystallization temperature of $380-440{ }^{\circ} \mathrm{C}[11]$.

X-ray diffraction (XRD) was performed on the as-cast and annealed ribbons. The XRD scans (Fig. 1) showed no evidence of bulk crystalline formation. Bulk magnetic properties were measured by vibrating sample magnetometry (VSM) with a Helmholtz field applied along the ribbon length. MOKE microscopy was conducted to image the domain structure on the surface of the ribbons. Longitudinal magneto-optical Kerr effect (L-MOKE) hysteresis loops were taken using a homemade setup with a $5 \mathrm{~mW}(655 \mathrm{~nm})$ laser source with a spot size $\sim 1.5 \times 1.5 \mathrm{~mm}^{2}$; about nine times larger than the field of view of the microscope.

Magneto-impedance was measured as a function of applied field $\left(H_{\max }=300 \mathrm{Oe}\right)$ along the length of the ribbon at high frequency (100-1000 MHz) using an HP 4191A impedance analyzer. The HP 4191A determines the complex reflection coefficient, $\Gamma$, of a high frequency signal sent down a terminated transmission line. An airline [17] made of a $2 \mathrm{~cm}$ section of ribbon suspended over a $\mathrm{Cu}$ ground plane served as the transmission line, which was terminated with a $50 \Omega$ standard 
that matches the input impedance of the analyzer. The complex reflection coefficient at the beginning of the ribbon airline was measured and converted to complex impedance $(\boldsymbol{Z})$ by

$$
Z=50 \frac{1-\Gamma}{1+\Gamma}=R+\mathrm{j} X
$$

where $R$ is the resistance, $X$ is the reactance, and $j$ is the imaginary unit. In this work, the magnetoimpedance ratio is defined as

$$
\frac{\Delta \mathrm{Z}}{\mathrm{Z}}(\%)=\frac{\mathrm{Z}(\mathrm{H})-\mathrm{Z}(\mathrm{H}=0)}{\mathrm{Z}(\mathrm{H}=0)} \times 100
$$

where $Z(H)$ is the impedance (or resistance, reactance) at field $H$. The impedance ratio is normalized by $Z(H=0)$.

Inductance formalism is generally used to connect complex impedance measurements directly with ac magnetic phenomena [18]. The transformation that takes place between $\boldsymbol{Z}$ and complex inductance, $\boldsymbol{L}$, is simple but leads to a crossover between the real and imaginary components. Therefore the real part of $Z$ is proportional to the imaginary part of the inductance, and vice versa. The complex inductance, in turn, is directly proportional to the complex permeability by a scalar geometrical factor. Thus, $R$ is related to the imaginary component and $X$ to the real component of the complex permeability. Furthermore, the nature of the ac magnetization process can be interpreted by the examination of the real and imaginary parts of the permeability, where, at frequencies greater than $100 \mathrm{MHz}$, the real part represents reversible rotation processes (imaginary part of the impedance) and the imaginary part represents dissipative processes such as the rotation of pinned domain spins.

\section{Results and Discussion}

Several MOKE microscopy images were made on the air side of as-cast $\mathrm{Nb0}$ and $\mathrm{Nb} 4$ ribbons along the ribbon length. Selected MOKE images are presented in Fig. 2 (a,c). For both 
compositions, the inhomogeneous distribution of quenched-in stresses from fabrication gives rise to a complicated domain structure. The $\mathrm{Nb} 4$ ribbon sample in particular had layered domain patterns where tightly-patterned regions of high anisotropy overlay wide planar domains. These fingerprintlike domain patterns are evidence of perpendicular magnetic anisotropy with respect to the ribbon surface [19]. The rounded L-MOKE hysteresis loops of the as-cast ribbons (dashed lines in Fig. 2 $(\mathbf{e}, \mathbf{f}))$ also reflect a large distribution of in-plane anisotropy near the surface.

The surface domain structure changed dramatically with the $3 \mathrm{~h}$ Joule annealing (JA) treatment. The Nb0 and Nb4 ribbon, Fig. 2 (a,c), both developed wide planar domains as a result. The absence of the 'fingerprint'-type domain patterns indicates that the JA treatment reduced the layered stresses quenched in during melt spinning. Interestingly, the Nb0 ribbon sample developed stripe-like domains along the ribbon axis, while the $\mathrm{Nb} 4$ sample developed an anisotropy $\sim 30^{\circ}$ from the transverse axis. The hysteresis loops, measured by a separate MOKE setup, show strikingly different switching behavior at the surface as a result of the $3 \mathrm{~h} \mathrm{JA}$ treatment. The annealed $\mathrm{Nb} 0$ ribbon sample developed a square hysteresis loop clearly indicating the longitudinal easy axis found in the microscopy images. The hysteresis loop of the annealed $\mathrm{Nb} 4$ ribbon shows a hard axis magnetic behavior, confirming the microscopy images showing the domain easy axis roughly $\sim 60^{\circ}$ away from the longitudinal measuring axis.

Bulk magnetometry from VSM (Fig. 3 (a)) shows both Nb0 and Nb4 compositions with nearly equal and vanishing coercivities and characteristic soft ferromagnetic behavior. The saturation magnetization $\left(M_{\mathrm{S}}\right)$ is lower for the $\mathrm{Nb} 4$ ribbon, which can negatively impact the MI response, especially at lower frequencies where the skin depth reaches deep into the bulk. From Fig. 3 (b) it can be seen that after a $3 \mathrm{~h}$ and $500 \mathrm{~mA}$ JA treatment, the bulk coercivity of the $\mathrm{Nb} 0$ composition increased from $H_{\mathrm{c}}=0.22$ Oe to $H_{\mathrm{c}}=0.65 \mathrm{Oe}$ and the $\mathrm{Nb} 4$ ribbon increased slightly from $H_{\mathrm{c}}=0.25$ Oe to $H_{\mathrm{c}}=0.41$ Oe. Overall, there is little indication of the bulk magnetic properties 
being affected by the JA technique. This could imply that the bulk of the ribbon did not reach temperatures high enough to begin bulk crystallization.

High-frequency MI was examined to probe the field dependence of the ac rotational (transverse) permeability near the surface of the ribbons. The real and imaginary components of the complex impedance were measured as a function of applied dc field at frequencies ranging from 100 to $1000 \mathrm{MHz}$. At these excitation frequencies, the ac magnetic field penetrates about 1-2 orders of magnitude deeper than the light probe in MOKE experiments and covers a much larger surface area. The field dependence of the MI ratios (Fig. 4) displays a typical high-frequency double-peak behavior; an assurance that there is negligible contribution to the ac permeability from domain wall motion. Thus, the increase in MI ratio from $H_{\mathrm{DC}} \sim 0$ is attributed to the decrease in skin depth from a rise in ac rotational permeability transverse to the current direction (i.e. along the ac field, $h_{\mathrm{ac}}$, direction). The increase in ac rotational permeability with $H_{\mathrm{DC}}$ can be understood by considering the competition between $H_{\mathrm{DC}}$ and the anisotropy field, $H_{\mathrm{k}}$, of a single domain with uniaxial anisotropy. Increasing $H_{\mathrm{DC}}$ can compensate the anisotropy field, which makes the ac rotational magnetization process is easier, thus increasing the rotational permeability until it reaches a maximum at a field close to $H_{\mathrm{k}}$. As the $H_{\mathrm{DC}}$ exceeds $H_{\mathrm{k}}$, more domain magnetizations couple to $H_{\mathrm{DC}}$, thereby lowering the ac rotational permeability causing the decrease in the MI ratios.

The magneto-resistance, magneto-reactance, and magneto-impedance ratios $(\Delta R / R, \Delta X / X, \&$ $\Delta Z / Z$ ) at $100 \mathrm{MHz}, 400 \mathrm{MHz}$, and $1000 \mathrm{MHz}$ of the as-cast ribbons are presented in Fig. 4 (a-i) (dashed lines). Examining the first column of Fig. 4, all the ratios of the as-cast $\mathrm{Nb0}$ and $\mathrm{Nb} 4$ ribbons have similar field dependent behavior, e.g. similar $H_{\mathrm{k}}$ values and anisotropy distributions, at the lowest measured frequency of $100 \mathrm{MHz}$. As the ac frequency is increased, the MI probes closer to the surface of the ribbon and a greater distinction between the different compositions of ribbon 
are apparent. At $400 \mathrm{MHz}$, second column of Fig. 4, the $\Delta R / R$ and $\Delta X / X$ ratios of the $\mathrm{Nb} 4$ ribbon begin to overtake those of the $\mathrm{Nb} 0$ ribbon, especially in the anisotropy field regime. At the highest measured frequency of $1000 \mathrm{MHz}$, third column, the similarities between the $\mathrm{Nb} 0$ and $\mathrm{Nb} 4$ ratios continue to diverge at $H_{\mathrm{DC}}$ fields much lower than $H_{\mathrm{k}}$.

The MI ratios at $100 \mathrm{MHz}$ of each ribbon after $3 \mathrm{~h}$ annealing (Fig. 4 (a-c), solid lines) show that the JA protocol had an impact on the MI of both compositions of ribbon. The $\Delta R / R$ ratio of annealed $\mathrm{Nb0}$ ribbon (black line) increases significantly from the as-cast state, while the $\Delta X / X$ ratio shows only a slight improvement. As compared to the $\mathrm{Nb} 0$ ribbon, the annealed $\mathrm{Nb} 4$ composition shows a more marked improvement in both ratios as a result of the JA treatment, especially in $\Delta X / X$. Considering the connection between the reactance, $X$, and real part of the permeability via inductance formalism, the enhancement of $\Delta X / X$ by the JA treatment had a greater impact on the reversible ac permeability of the ribbons with the growth inhibitor $\mathrm{Nb}$. This is connected to the domain structure depicted in MOKE images of the annealed ribbons in Fig. 2 (b,d), which shows the development of stripe-like domains at different orientations with respect to the transverse direction. The preferential direction of the surface anisotropy for the $\mathrm{Nb} 4$ ribbon is aligned much closer ( 30 degrees) to the transverse direction, which supports reversible ac rotational permeability.

The maximum change in MI, $[\Delta Z / Z]_{\max }$, of the field-dependent $\Delta Z / Z$ curves (Fig. 4) occurs when the transverse permeability $\mu_{\mathrm{T}}\left(H_{\mathrm{DC}}\right)$ takes its highest value. The frequency dependence of $[\Delta Z / Z]_{\max }$ is presented in Fig. 5 (a). The JA treatment increased $[\Delta Z / Z]_{\max }$ of both ribbons over the entire test frequency regime as compared to their as-cast counterparts. In the higher frequency region, however, the improved $[\Delta Z / Z]_{\max }$ of the sample with growth inhibitor $\mathrm{Nb}$ persists at high frequencies where the values of $[\Delta Z / Z]_{\max }$ of the ribbon without $\mathrm{Nb}$ approach the as-cast value. This also implies the JA had a greater impact on the ac transverse permeability of the ribbon with 
growth inhibitor $\mathrm{Nb}$. The JA treatment also caused a shift of the $[\Delta Z / Z]_{\max }$ peak to lower frequencies as compared to the as-cast state. A broad peak in $[\Delta Z / Z]_{\max }$ can be seen near 200 and $400 \mathrm{MHz}$ for the as-cast $\mathrm{Nb0}$ and $\mathrm{Nb} 4$ ribbon, respectively, where upon annealing the peaks shift to $<100 \mathrm{MHz}$ and $200 \mathrm{MHz}$, respectively.

Another interesting feature of Fig. 5 (a) concerns a cross over that takes place between the $[\Delta Z / Z]_{\max }$ of the $\mathrm{Nb} 0$ and $\mathrm{Nb} 4$ compositions, an indication that at lower frequencies $\mathrm{Nb} 0$ has higher MI ratios. Indeed, as the MI probe frequency is decreased, the skin depth undoubtedly samples a larger portion of the ribbon and thus bulk magnetic properties have a greater contribution to the MI. This is further supported by the larger bulk $M_{\mathrm{S}}$ of the ribbon without the $\mathrm{Nb}$ growth inhibitor. Considering the consequence of the skin effect, as the frequency increases the bulk properties that once promoted MI at lower frequencies make less of an impact. Since it is clear from the neartransverse anisotropy of the annealed Nb4 ribbons that its $\mu_{\mathrm{T}}$, hence $[\Delta Z / Z]_{\max }$, is higher at larger excitation frequencies, it is possible that crossover could represent the skin depth where the near transverse surface anisotropy of $\mathrm{Nb} 4$ outweighs the higher bulk $M_{\mathrm{S}}$ of the $\mathrm{Nb} 0$ ribbon.

The corresponding field point of $[\Delta Z / Z]_{\max }$, defined as $H_{\mathrm{k}}$, is presented in Fig. 5 (b). The effect of annealing is obvious as the $H_{\mathrm{k}}$ values are greatly reduced across the excitation frequency range. This is linked to the increase in $[\Delta Z / Z]_{\max }$ since $H_{\mathrm{k}}$ is inversely proportional to $\mu_{T}$ when considering the simple case of reversible rotational magnetization process of a single domain with uniaxial anisotropy [20,21]. The $H_{\mathrm{k}}$ values for the as-cast ribbons (open symbols) are similar at low frequencies but noticeably diverge from each other near $500 \mathrm{MHz}$. Interestingly, upon annealing, the $H_{\mathrm{k}}$ values (closed symbols) of the two different ribbons have a similar behavior, however, now the divergence begins at a much lower frequency of $\sim 100 \mathrm{MHz}$.

\section{Conclusions}


The melt-spun Fe-rich FeCo alloy ribbons with and without crystallite growth inhibitor $\mathrm{Nb}$ were Joule annealed at low current intensity to avoid bulk crystallization. The effect of the $\mathrm{Nb}$ doping on the surface magnetic properties of the ribbons was investigated with MOKE and highfrequency MI. The surface of the $\mathrm{Nb} 0$ ribbon $(0$ at. $\% \mathrm{Nb}$ ) developed a longitudinal anisotropy as a result of the Joule annealing treatment, while the $\mathrm{Nb} 4$ ribbon (4 at. \% $\mathrm{Nb}$ ) developed an anisotropy $30^{\circ}$ from the transverse direction. The $H_{k}$ of both ribbons decreased after annealing, indicating an increase in transverse ac permeability. The near transverse anisotropy at the surface of $\mathrm{Nb} 4$ is evident in MI measurements by a higher $[\Delta Z / Z]_{\max }$ as compared to $\mathrm{Nb0}$ at frequencies greater than $200 \mathrm{MHz}$. A good understanding of the correlation between the magnetic domain structure and MI in soft ferromagnetic thin films [22,23] and microwires [24] has provided useful guidance on tailoring their MI properties for high-performance magnetic sensors, and our study presented in this article also serves the same purpose.

\section{Acknowledgements}

Research at USF was supported by the U.S. Department of Energy, Office of Basic Energy

Sciences, Division of Materials Sciences and Engineering under Award No. DE-FG02-07ER46438 (Structural characterization and magnetoimpedance studies). MHP also acknowledges the CASUSF grant. The Slovak group acknowledges the support of the projects APVV-0492-11 and ITMS 26220120035. 


\section{References}

[1] E.P. Harrison, G.L. Turney, H. Rowe, Electrical Properties of Wires of High Permeability, Nature. 135 (1935) 961-961. doi:10.1038/135961a0.

[2] M.H. Phan, H.X. Peng, Giant magnetoimpedance materials: Fundamentals and applications, Prog. Mater. Sci. 53 (2008) 323-420. doi:10.1016/j.pmatsci.2007.05.003.

[3] R.S. Beach, a. E. Berkowitz, Giant magnetic field dependent impedance of amorphous FeCoSiB wire, Appl. Phys. Lett. 64 (1994) 3652-3654. doi:10.1063/1.111170.

[4] L.V. Panina, K. Mohri, T. Uchiyama, M. Noda, K. Bushida, Giant magneto-impedance in Co-rich amorphous wires and films, IEEE Trans. Magn. 31 (1995) 1249-1260. doi:10.1109/20.364815.

[5] M. Heshmatzadeh, G.E. Bridges, G. Williams, High-frequency giant magnetoimpedance measurement and complex permeability behavior of soft magnetic co-based ribbons, IEEE Trans. Magn. 41 (2005) 2328-2333. doi:10.1109/TMAG.2005.852950.

[6] L.G.C. Melo, P. Ciureanu, A. Yelon, Permeability deduced from impedance measurements at microwave frequencies, J. Magn. Magn. Mater. 249 (2002) 337-341. doi:10.1016/S03048853(02)00555-3.

[7] M. Knobel, K.R. Pirota, Giant magnetoimpedance: Concepts and recent progress, J. Magn. Magn. Mater. 242-245 (2002) 33-40. doi:10.1016/S0304-8853(01)01180-5.

[8] A.M. Leary, P.R. Ohodnicki, M.E. Mchenry, Soft Magnetic Materials in High-Frequency , High-Power Conversion Applications, JOM. 64 (2012) 772-781. doi:10.1007/sll837-0120350-0.

[9] M.E. McHenry, M.A. Willard, D.E. Laughlin, Amorphous and nanocrystalline materials for 
applications as soft magnets, Prog. Mater. Sci. 44 (1999) 291-433. doi:10.1016/s00796425(99)00002-x.

[10] A. Chaturvedi, N. Laurita, A. Leary, M.H. Phan, M.E. McHenry, H. Srikanth, Giant magnetoimpedance and field sensitivity in amorphous and nanocrystalline $\left(\mathrm{Co}_{1-\mathrm{x}} \mathrm{Fe}_{\mathrm{x}}\right)_{89} \mathrm{Zr}_{7} \mathrm{~B}_{4}$ $(x=0,0.025,0.05,0.1)$ ribbons, J. Appl. Phys. 109 (2011) 5-7. doi:10.1063/1.3540403.

[11] K.J. Miller, A. Leary, S.J. Kernion, A. Wise, D.E. Laughlin, M.E. McHenry, et al., Increased induction in FeCo-based nanocomposite materials with reduced early transition metal growth inhibitors, J. Appl. Phys. 107 (2010) 09A316. doi:10.1063/1.3350900.

[12] S. Kernion, V. Keylin, J. Huth, M.E. McHenry, Secondary crystallization in $\left(\mathrm{Fe}_{65} \mathrm{Co}_{35}\right)_{79.5+\mathrm{x}} \mathrm{B}_{13} \mathrm{Nb}_{4-\mathrm{x}} \mathrm{Si}_{2} \mathrm{Cu}_{1.5}$ and $\left(\mathrm{Fe}_{65} \mathrm{Co}_{35}\right)_{83} \mathrm{~B}_{10} \mathrm{Nb}_{4} \mathrm{Si}_{2} \mathrm{Cu}_{1}$ nanocomposite alloys, J. Appl. Phys. 111 (2012) 07A329. doi:10.1063/1.3677830.

[13] G. Herzer, Soft-magnetic nanocrystalline materials, Scr. Metall. Mater. 33 (1995) 1741-1756. doi:10.1016/0956-716x(95)00397-e.

[14] S. Shen, V. DeGeorge, P. Ohodnicki, S. Kernion, V. Keylin, J.F. Huth, M.E. McHenry, Induced anisotropy in FeCo-based nanocomposites: Early transition metal content dependence, J. Appl. Phys. 115 (2014) 17A335. doi:10.1063/1.4867124.

[15] C. Miguel, A. Zhukov, J.J. del Val, J. González, Coercivity and induced magnetic anisotropy by stress and/or field annealing in Fe- and Co- based (Finemet-type) amorphous alloys, J. Magn. Magn. Mater. 294 (2005) 245-251. doi:10.1016/j.jmmm.2005.03.041.

[16] F. Johnson, H. Garmestani, S.Y. Chu, M.E. McHenry, D.E. Laughlin, Induced anisotropy in FeCo-based nanocrystalline ferromagnetic alloys (HITPERM) by very high field annealing, IEEE Trans. Magn. 40 (2004) 2697-2699. doi:10.1109/TMAG.2004.832278. 
[17] L. Brunetti, P. Tiberto, F. Vinai, A new measurement method for magneto-impedance in soft amorphous ferromagnets at microwave frequency, Sensors Actuators A Phys. 67 (1998) 8488.

[18] R. Valenzuela, Giant magnetoimpedance and inductance spectroscopy, J. Alloys Compd. 369 (2004) 40-42. doi:10.1016/j.jallcom.2003.09.057.

[19] A. Hubert, R. Schafer, Magnetic Domains, 1998. doi:10.1049/sqj.1970.0020.

[20] S.S. Yoon, S.C. Yu, G.H. Ryu, C.G. Kim, Effect of annealing on anisotropy field in $\mathrm{Fe}_{84} \mathrm{Zr}_{7} \mathrm{~B}_{8} \mathrm{Cu}_{1}$ amorphous ribbons evaluated by giant magnetoimpedance, J. Appl. Phys. 85 (1999) 5432.

[21] G.H. Ryu, S.C. Yu, C.G. Kim, S.S. Yoon, Evaluation of anisotropy field in amorphous $\mathrm{Fe}_{71+x} \mathrm{Nb}_{7} \mathrm{~B}_{22-\mathrm{x}}$ alloys by GMI measurement, J. Magn. Magn. Mater. 215-216 (2000) 359361. doi:10.1016/S0304-8853(00)00158-X.

[22] G.V. Kurlyandskaya, L. Elbaile, F. Alves, B. Ahamada, R. Barrué, A.V. Svalov, V.O. Vas'kovskiy, Domain structure and magnetization process of a giant magnetoimpedance geometry $\mathrm{FeNi} / \mathrm{Cu} / \mathrm{FeNi}(\mathrm{Cu}) \mathrm{FeNi} / \mathrm{Cu} / \mathrm{FeNi}$ sensitive element, J. Phys. Condens. Matter. 16 (2004) 6561-6568. doi:10.1088/0953-8984/16/36/021.

[23] D. Garcia, G.V. Kurlyandskaya, Induced anisotropy, magnetic domain structure and magnetoimpedance effect in CoFeB amorphous thin films, J. Magn. Magn. Mater. 191 (1999) 339-344. doi:10.1016/S0304-8853(98)00262-5.

[24] M. Ipatov, A. Chizhik, V. Zhukova, J. Gonzalez, A. Zhukov, Correlation of surface domain structure and magneto-impedance in amorphous microwires, J. Appl. Phys. 109 (2011) 1-7. doi:10.1063/1.3596808. 


\section{Figure captions}

Figure 1. X-ray diffraction of the as-quenched and annealed ribbons.

Figure 2. MOKE microscopy images of (a) Nb0 as-cast (b) annealed 3 h @ 500 mA, (c) Nb4 ascast, and (d) Nb4 annealed 3 h @ 500 mA ribbons. Panels (e) and (f) are longitudinal MOKE hysteresis loops taken on a different section of ribbon from (a)-(d).

Figure 3. (a) $M-H$ loops from vibrating sample magnetometry at room temperature on $6 \times 1 \mathrm{~mm}^{2}$ sections of ribbon before and after $3 \mathrm{~h}$ JA treatment. The magnetic field was applied along the ribbon axis. (b) Zoom in of the vanishing coercivity of all samples before and after $3 \mathrm{~h}$ JA treatment.

Figure 4. The magnetoresistance $(\Delta R / R)$, magnetoreactance $(\Delta X / X)$ and magnetoimpedance $(\Delta Z / Z)$ ratios measured at $f=100 \mathrm{MHz}(\mathrm{a}-\mathrm{c}), 400 \mathrm{MHz}(\mathrm{d}-\mathrm{f})$, and $1000 \mathrm{MHz}$ (g-i). As quenched ribbons are dashed lines and solid lines are after a $3 \mathrm{~h}$ JA treatment. Note only increasing $H_{\mathrm{DC}}$ field branches are shown for clarity.

Figure 5. (a) Maximum change in $\Delta Z / Z$ as a function of excitation frequency for the as-cast (open symbols) and annealed ribbons (closed symbols). (b) The anisotropy field $\left(H_{\mathrm{k}}\right)$ as a function of excitation frequency. The open symbols are as-cast ribbons and closed symbols are after a $3 \mathrm{~h}$ JA treatment. 


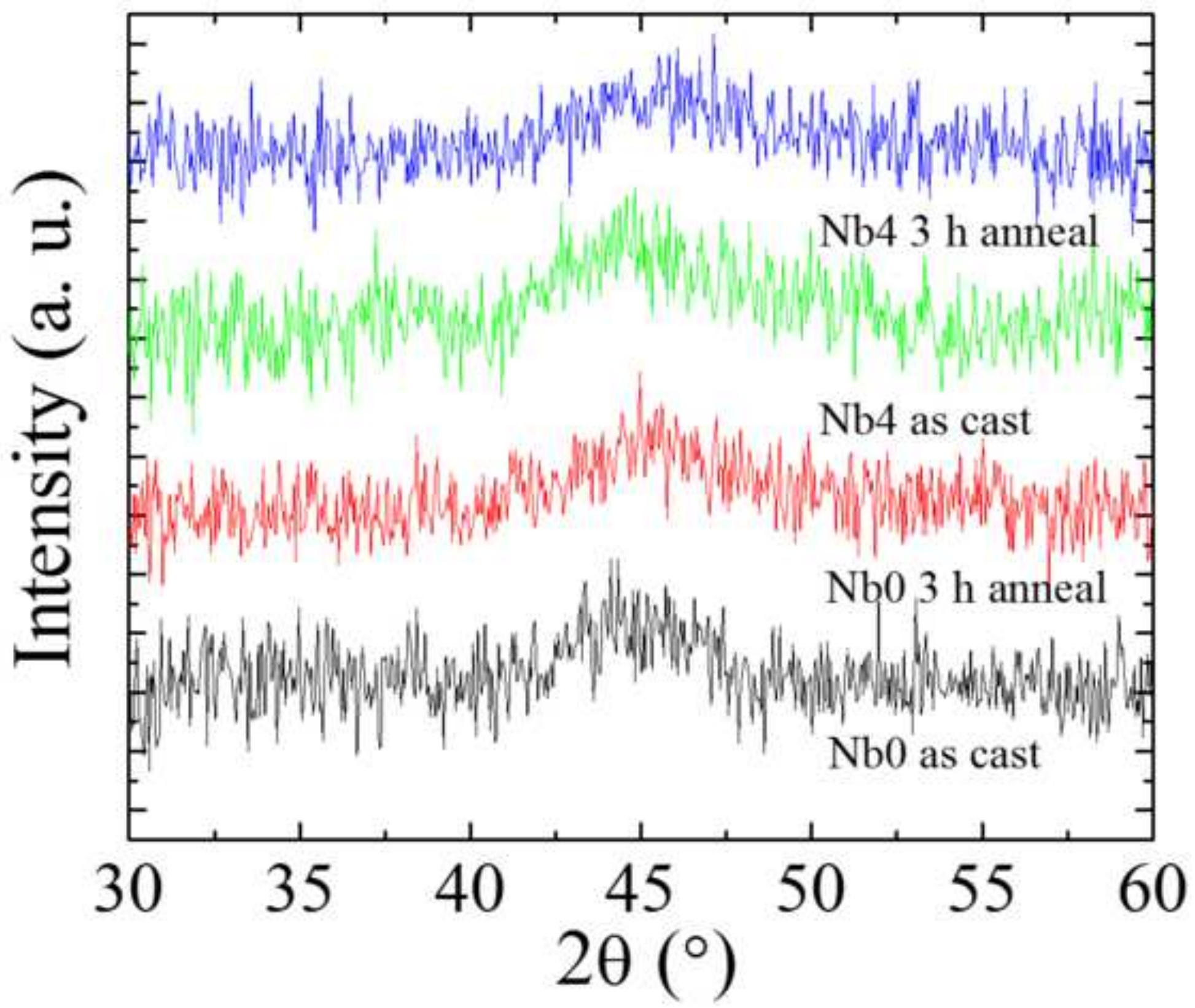



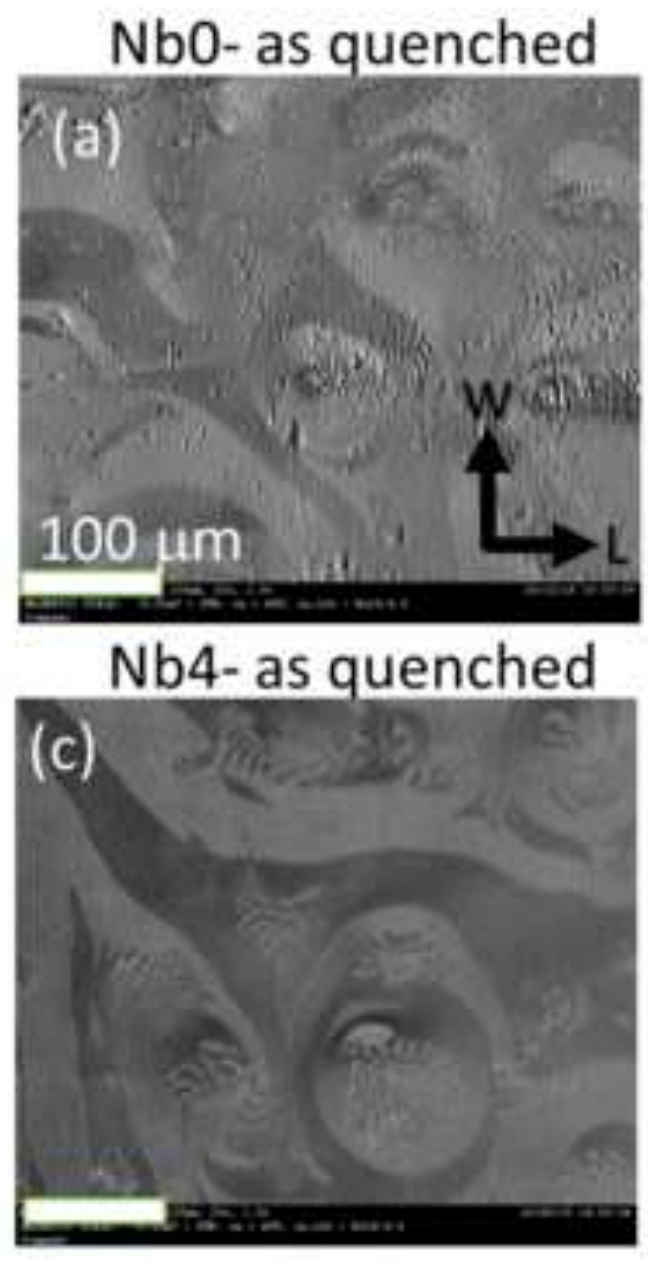
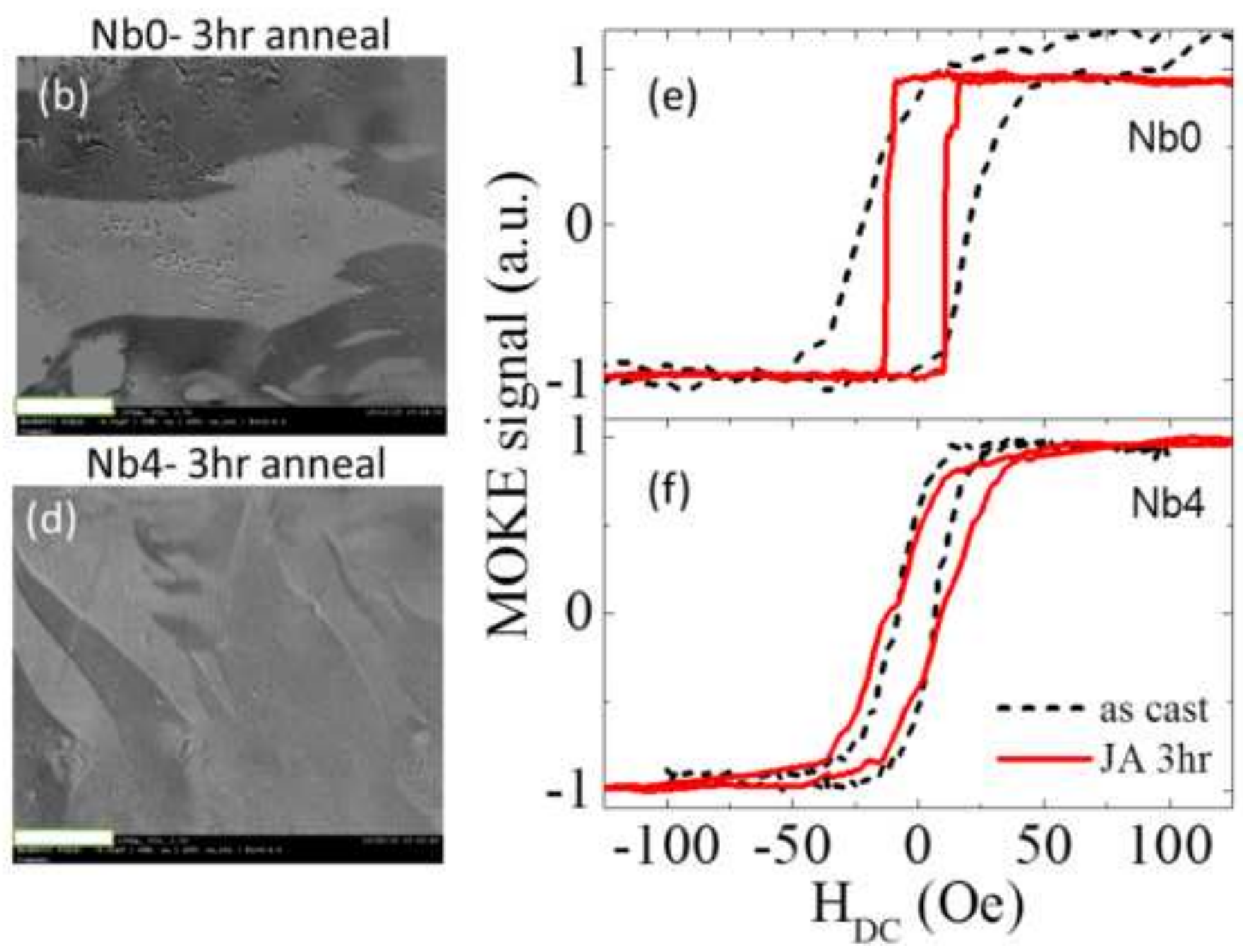


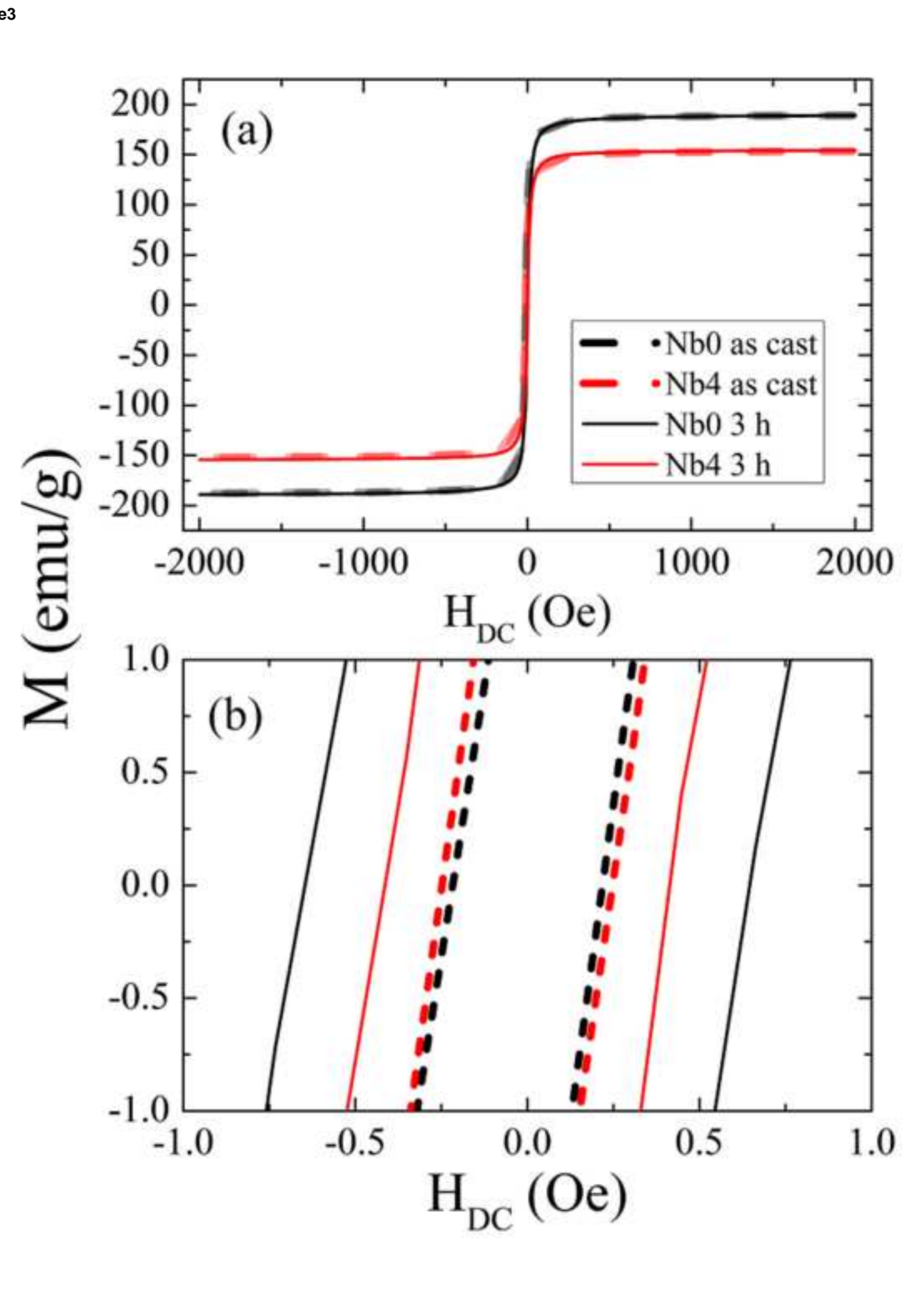

Figure3

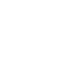

\section{.}

.
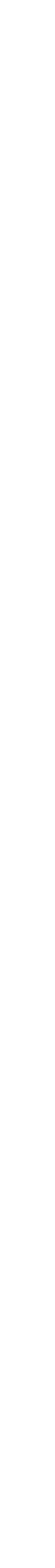


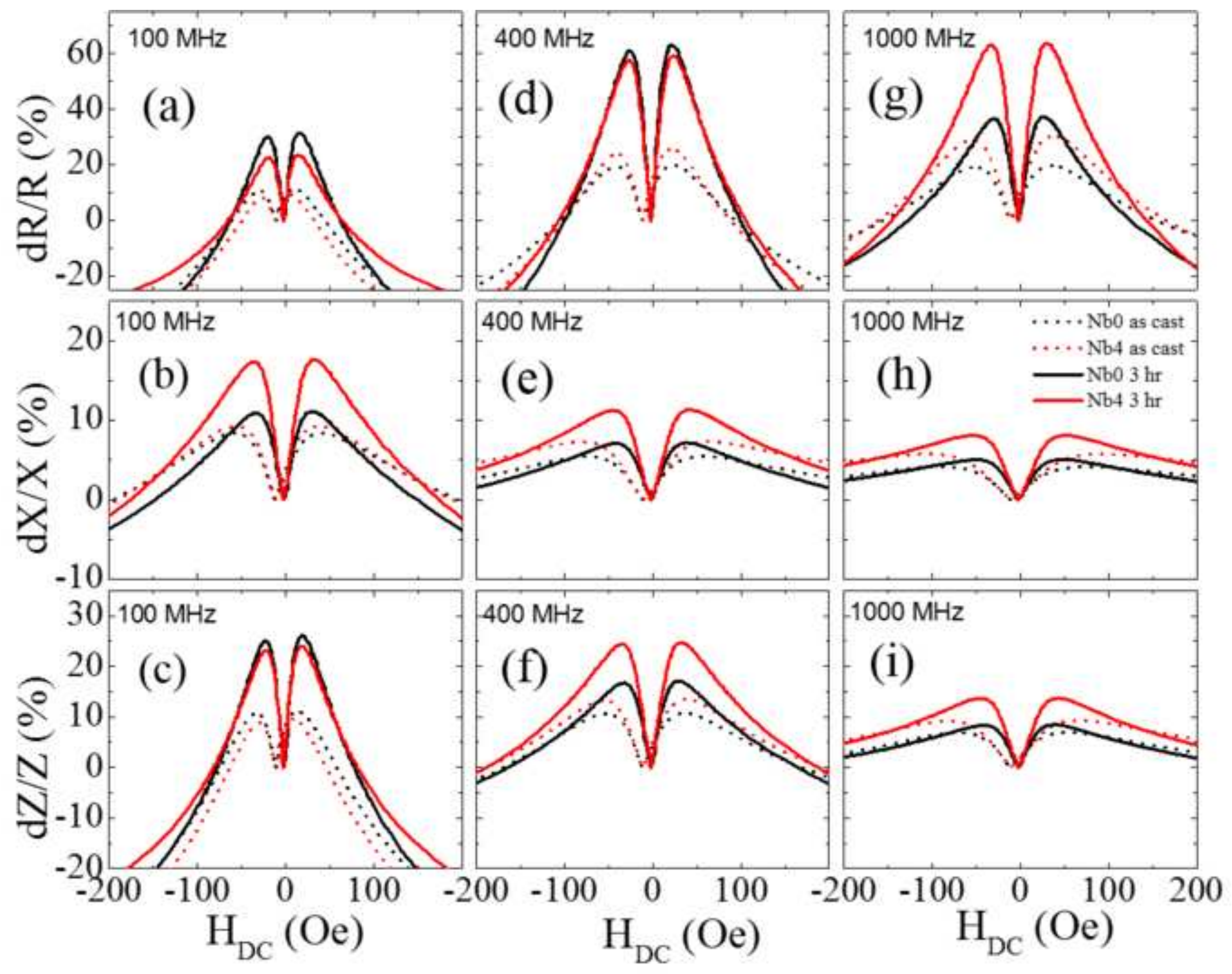



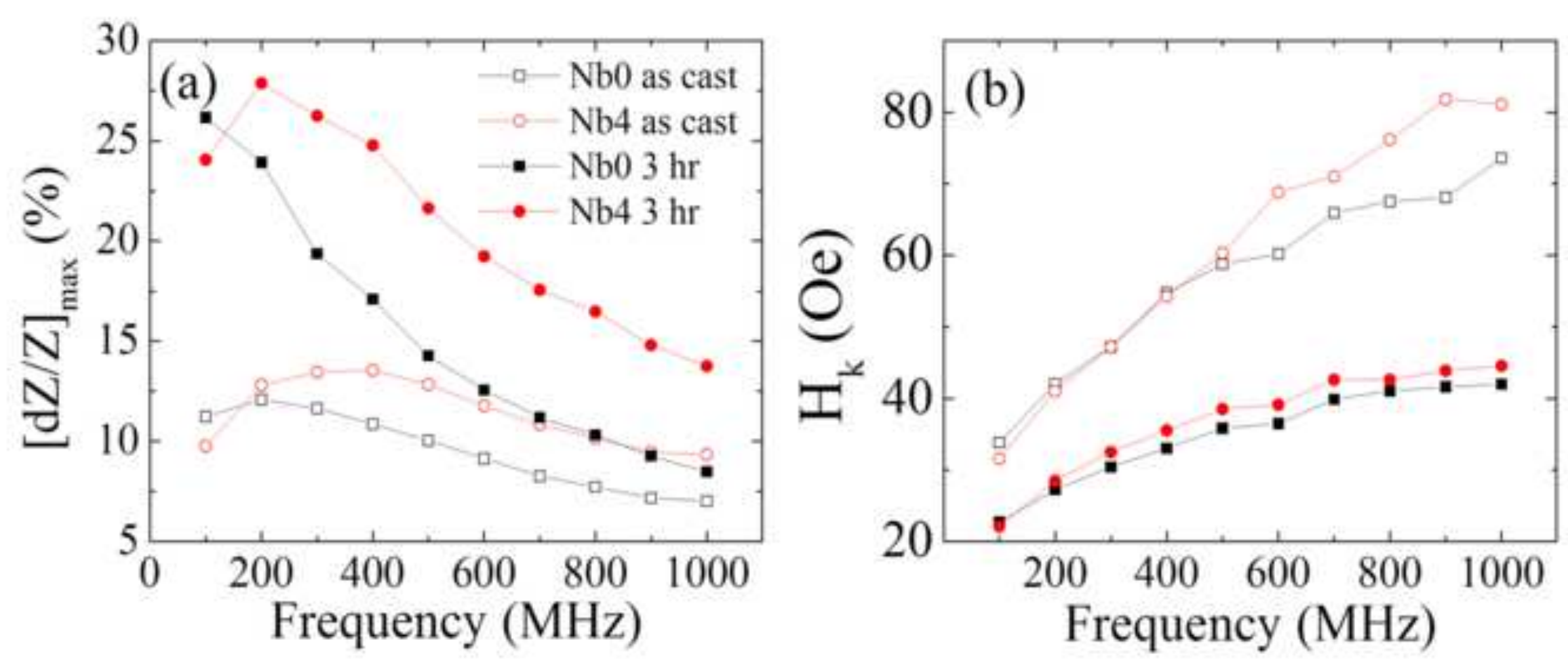